\title{
Reliability of the density functional approximation to describe the charge transfer and electrostatic complexes involved in the modeling of organic conducting polymers
}

\author{
Carlos Alemán, ${ }^{1, *}$ David Curcó, ${ }^{2}$ and Jordi Casanovas ${ }^{3, \dagger}$ \\ ${ }^{1}$ Departament d'Enginyeria Química, E.T.S. d'Enginyers Industrials de Barcelona, Universitat Politècnica de Catalunya, \\ Diagonal 647, Barcelona E-08028, Spain \\ ${ }^{2}$ Departament d'Enginyeria Química, Facultat de Química, Universitat de Barcelona, Marí i Franques 1, Barcelona E-08028, Spain \\ ${ }^{3}$ Departament de Química, Escola Politècnica Superior, Universitat de Lleida, c/ Jaume II No. 69, Lleida E-25001, Spain
}

(Received 30 March 2005; revised manuscript received 13 May 2005; published 17 August 2005)

\begin{abstract}
Both the intermolecular interaction energies and the geometries for $M \cdots$ thiophene, $M^{\cdots}$ pyrrole, $M^{n+}$.. thiophene, and $M^{n+} \ldots$ pyrrole (with $M=\mathrm{Li}, \mathrm{Na}, \mathrm{K}, \mathrm{Ca}$, and $\mathrm{Mg}$; and $M^{n+}=\mathrm{Li}^{+}, \mathrm{Na}^{+}, \mathrm{K}^{+}, \mathrm{Ca}^{2+}$, and $\mathrm{Mg}^{2+}$ ) have been estimated using four commonly used density functional theory (DFT) methods: B3LYP, B3PW91, PBE, and MPW1PW91. Results have been compared to those provided by HF, MP2, and MP4 conventional $a b$ initio methods. The PBE and MPW1PW91 are the only DFT methods able to provide a reasonable description of the $M \cdots \pi$ complexes. Regarding $M^{n+} \cdots \pi$ complexes, the four DFT methods have been proven to be adequate in the prediction of these electrostatically stabilized systems, even though they tend to overestimate the interaction energies.
\end{abstract}

DOI: 10.1103/PhysRevE.72.026704

PACS number(s): 31.70.-f, 31.15.Ew

\section{INTRODUCTION}

The interactions of $\pi$-conjugated molecules, such as pyrrole and thiophene, with metallic atoms either in neutral or positively charged states ( $M$ and $M^{n+}$, respectively) are essential in the fields of polymer and material sciences. Thus $M \cdots \pi$ and $M^{n+\cdots} \pi$ interactions are involved in the doping of organic conducting polymers. Within this context, model complexes containing such interactions have been satisfactorily used to model the doping of organic conducting polymers through quantum mechanical calculations [1-7].

Density functional theory (DFT) offers a very interesting alternative to conventional ab initio methods, such as MP2 and MP4, for describing the interaction of metallic species with $\pi$-conjugated molecules. The most reliable advantage of DFT with respect to these sophisticated $a b$ initio methods is that the former includes correlation effects at a lower computational cost, i.e., the cost is $N^{3}, N^{5}$, and $N^{7}$ for DFT, MP2, and MP4, respectively, where $N$ is the number of basis functions. However, the ability of DFT calculations to describe noncovalent interactions has been shown to depend on the form of the functional. In the last decade, the suitability of the more popular functionals to predict intermolecular interaction energies and geometries for hydrogen bonded [8-11], van der Waals [12-14], and aromatic $\pi-\pi$ [15-17] complexes has been checked.

In this work we evaluate the performance of the B3LYP, B3PW91, PBE, and MPW91PW91 functionals to describe both $M \cdots \pi$ and $M^{n+\cdots} \pi$ interactions. Calculations have been performed on systems formed by thiophene (Thp) and pyrrole (Py) interacting with $M=\mathrm{Li}, \mathrm{Na}, \mathrm{K}, \mathrm{Ca}$, and $\mathrm{Mg}$; and $M^{n+}=\mathrm{Li}^{+}, \mathrm{Na}^{+}, \mathrm{K}^{+}, \mathrm{Mg}^{2+}$, and $\mathrm{Ca}^{2+}$. Results provided by DFT calculations have been compared to those obtained at the HF, MP2, and MP4 levels.

\footnotetext{
*Email address: carlos.aleman@upc.edu

†Email address: jcasanovas@quimica.udl.es
}

\section{METHODS}

All $a b$ initio and DFT calculations were performed using the GAUSSIAN 98 [18] program. Ab initio calculations were carried out at the HF, MP2, and MP4 [19] levels of theory. DFT calculations were performed using four combinations: the Becke's three-parameter hybrid functional (B3) [20] with the Lee, Yang, and Parr (LYP) [21] expression for the nonlocal correlation (B3LYP); the same functional with the nonlocal correlation provided by Perdew and Wang (B3PW91) [22]; the approximation of Perdew, Burke, and Ernzerhof (PBE) $[23,24]$, which is a simpler version of PW91; and the modified Perdew-Wang exchange [25] with the PW91 gradient-corrected correlation [22] (MPW1PW91). Calculations on closed-shell and open-shell systems were done considering the restricted and unrestricted quantum-chemical formalisms, respectively. Geometry optimizations were performed at the HF, MP2, B3LYP, B3PW91, PBE, and MPW1PW91 levels of theory, while MP4 energies (including all single, double, triple, and quadruple excitations) were derived from single point calculations on geometries optimized at the MP2 level. The 6-31+ $\mathrm{G}(d, p)$ basis set [26] was used in all the $a b$ initio and DFT calculations. Frequency analyses were carried out to verify the nature of the minimum state of all the stationary points located during geometry optimizations.

The interaction energies $\left(\Delta E_{\text {int }}\right)$ were calculated as the difference between the energy of the optimized complex and the sum of the energies of the isolated fragments ( $\pi$-conjugated ring and metal atom). The counterpoise method [27] (CP) was applied to correct the basis set superposition error (BSSE) in the interaction energies.

In order to compare the molecular geometries predicted by the different computational methods, the same starting arrangement was considered for all the complexes. This consists of the metallic specie $\left(M\right.$ or $\left.M^{n+}\right)$ located above the center of the molecular ring (Fig. 1). 


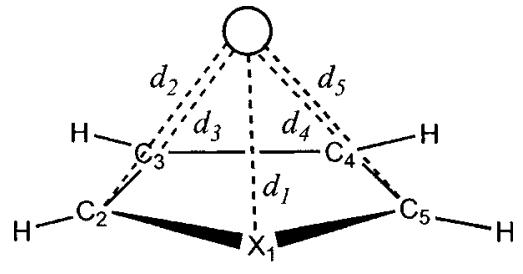

$$
\begin{gathered}
d^{2,5}=\frac{d_{2}+d_{5}}{2} \quad d^{3,4}=\frac{d_{3}+d_{4}}{2} \\
\mathrm{X}_{1}=\mathrm{S}(\mathrm{Thp}) \text { or N-H (Py) }
\end{gathered}
$$

FIG. 1. Structure of the complexes investigated in this work. The geometric parameters $\left(d_{1}, d^{2,5}\right.$, and $\left.d^{3,4}\right)$ used to compare the geometries provided by the different methods (see text) are defined.

\section{RESULTS AND DISCUSSION}

\section{A. Interaction energies}

The interaction energies with $\mathrm{CP}$ corrections calculated for $M \cdots$ Thp and $M \cdots$ Py charge transfer complexes are listed in Table I. As expected MP2 and MP4 interaction energies are remarkably similar in all cases. The largest difference $(2.8 \mathrm{kcal} / \mathrm{mol})$ corresponds to the $\mathrm{Li}$ - Thp complex, where the inclusion of electron correlation effects up to fourth-order results in a counterbalancing of the excess of stability achieved at the MP2 level. For the rest of the complexes MP2 interaction energies are within $0.3 \mathrm{kcal} / \mathrm{mol}$ of MP4. Comparison between the MP4 and HF energies points out the limitations of the latter method indicating that inclusion of electron correlation effects is essential. Thus the HF method provides a notable underestimation of the interaction energies for $M \cdots P y$ complexes with $M=\mathrm{Li}, \mathrm{Na}$, and $\mathrm{K}$;

TABLE I. Interaction energies ${ }^{\mathrm{a}}$ (in $\mathrm{kcal} / \mathrm{mol}$ ) calculated for charge transfer complexes $M \cdots$ thiophene and $M \cdots$ pyrrole. In every case the level of theory used to calculate the interaction energy is identical to that used to obtain the optimized geometry with exception of the MP4 level, where the MP2 optimized geometry was used $(\text { see Sec. II })^{\mathrm{b}}$

\begin{tabular}{crrrrrrr}
\hline \hline$M$ & MP2 & MP4 & HF & B3LYP & B3PW91 & PBE & MPW1PW91 \\
\hline \multicolumn{7}{c}{$M \cdots$ Thiophene } \\
$\mathrm{Li}$ & -11.8 & -9.0 & -8.7 & -19.8 & -19.5 & -19.5 & -20.7 \\
$\mathrm{Na}$ & -0.9 & -1.1 & 0.0 & -0.2 & -0.4 & -1.4 & -1.0 \\
$\mathrm{~K}$ & -1.4 & -1.3 & 0.0 & -0.2 & -0.3 & -1.6 & -1.1 \\
$\mathrm{Mg}$ & -1.6 & -1.3 & 0.0 & 0.0 & 0.0 & -1.5 & -0.7 \\
$\mathrm{Ca}$ & -1.5 & -1.2 & 0.0 & 0.0 & -0.1 & -1.2 & -0.9 \\
\hline & & & \multicolumn{7}{c}{$M \cdots$ Pyrrole } & & \\
$\mathrm{Li}$ & -4.6 & -4.8 & -2.5 & -4.4 & -4.8 & -6.8 & -6.5 \\
$\mathrm{Na}$ & -2.4 & -2.6 & -0.7 & -1.1 & -1.9 & -3.2 & -2.7 \\
$\mathrm{~K}$ & -3.3 & -3.1 & -1.6 & -1.8 & -2.0 & -3.5 & -3.0 \\
$\mathrm{Mg}$ & -2.3 & -2.0 & -0.1 & -0.1 & -0.8 & -2.3 & -1.7 \\
$\mathrm{Ca}$ & -2.5 & -2.3 & -0.1 & -0.4 & -1.5 & -3.2 & -2.6 \\
\hline \hline
\end{tabular}

${ }^{\mathrm{a}}$ The BSSE was corrected using the CP method.

${ }^{\mathrm{b}}$ All calculations were carried out using the $6-31+\mathrm{G}(d, p)$ basis set. while no stabilization was achieved for $M \cdots$ Py with $M$ $=\mathrm{Mg}, \mathrm{Ca}$ and $M \cdots$ Thp with $M \neq \mathrm{Li}$, i.e., geometry optimization led to a separation of the fragments. Interestingly, the $\mathrm{Li}$ - .Thp was the only complex satisfactorily reproduced at the HF level.

On the other hand, comparison between DFT and MP4 interaction energies reveals notable discrepancies, which in turn depend on the functional used in the former calculations. The B3LYP and B3PW91 methods, especially the former one, underestimate the stability of $M \cdots$ Thp with $M$ $=\mathrm{Na}, \mathrm{K}$ and $M \cdots$ Py with $M \neq \mathrm{Li}$, and neglect the existence of $M \cdots$ Thp with $M=\mathrm{Mg}$, Ca. Furthermore, these functionals reproduce satisfactorily the interaction energy of the $\mathrm{Li} \cdot \cdots \mathrm{Py}$ complex, while that of $\mathrm{Li} \cdot$. Thp is drastically overestimated. The PBE and MPW1PW91 methods provide the best DFT estimates. Thus the interaction energies predicted these approximations for $M \cdots$ Thp and $M \cdots$ Py complexes with $M$ $\neq \mathrm{Li}$ are in good agreement with the MP4 ones. However, the values predicted for $\mathrm{Li} \cdot$. Thp and $\mathrm{Li} \cdot$.Py are overestimated by $65-75 \%$ and $41-48 \%$, respectively. The overall of these results indicate that the PBE and MPW1PW91 are the better DFT methods adequate to describe the energetics involved in $M \cdots \pi$ complexes.

The interaction energies computed at the $a b$ initio and DFT levels for $M^{n+} \ldots$ Thp and $M^{n+}$.. Py complexes are shown in Table II. Comparison of results listed in Tables I and II shows that the interaction energies are much more negative when the metal atom is ionized. Thus $M^{n+} \ldots$ Thp and $M^{n+} \ldots$ Py are dominated by a strong electrostatic interaction between the $\pi$ cloud of the heterocycle and the positively charged atom. Accordingly, at the highest level of theory the interaction energies range from -30.8 to $-13.6 \mathrm{kcal} / \mathrm{mol}$ and from -37.2 to $-18.0 \mathrm{kcal} / \mathrm{mol}$ when a singly charged atom $M^{+}$is complexed with Thp and Py, respectively. These values are one order of magnitude more favorable than those shown in Table I for $M \cdots$ Thp and $M \cdots$ Py. On the other hand, when the metallic specie presents two positive charges, as in $M^{2+} \cdot \mathrm{Thp}(\mathrm{Py})$ complexes, the interaction energy amounts to $-104.2(-113.1)$ and $-59.6(-68.8) \mathrm{kcal} / \mathrm{mol}$ for $\mathrm{M}^{2+}=\mathrm{Mg}^{+2}$ and $\mathrm{Ca}^{2+}$, respectively.

Analysis of electron correlation effects in Table II indicates that the change from the MP2 to MP4 level leads to very small changes in the interaction energy (typically around $0.4 \mathrm{kcal} / \mathrm{mol}$, i.e., $\sim 1 \%$ ) suggesting a good convergence. On the other hand, the HF method tends to overestimate the strength of the interaction in these electrostatically stabilized complexes by only $\sim 2.5 \%$, which is a very satisfactory result. Finally, the four functionals investigated provide a reasonable description of the complexes containing a metal cation, although in all cases the strength of the interaction is overestimated with respect to the $a b$ initio values. This overestimation is about 9\% for both B3LYP and B3PW91 functionals, while the MPW1PW91 and, particularly, the PBE methods provide the more negative values of the interaction energies, i.e., the overestimation is of about $11 \%$. The similarity between B3LYP and B3PW91 results combined with the enhancement produced by the PBE and MPW1PW91 methods suggest that the exchange functionals are responsible for the DFT overestimation. 
TABLE II. Interaction energies ${ }^{\mathrm{a}}$ (in $\mathrm{kcal} / \mathrm{mol}$ ) calculated for complexes $M^{n+\ldots}$ thiophene and $M^{n+}$...pyrrole. In every case the level of theory used to calculate the interaction energy is identical to that used to obtain the optimized geometry with exception of the MP4 level, where the MP2 optimized geometry was used (see Sec. II). ${ }^{\mathrm{b}}$

\begin{tabular}{cccccccc}
\hline \hline$M^{n+}$ & MP2 & MP4 & HF & B3LYP & B3PW91 & PBE & MPW1PW91 \\
\hline \multicolumn{7}{c}{$M^{n+.}$ Thiophene } \\
$\mathrm{Li}^{+}$ & -31.2 & -30.8 & -32.2 & -34.3 & -33.4 & -35.3 & -34.7 \\
$\mathrm{Na}^{+}$ & -19.6 & -19.4 & -20.4 & -21.9 & -21.1 & -22.9 & -22.2 \\
$\mathrm{~K}^{+}$ & -14.0 & -13.6 & -13.1 & -13.9 & -13.6 & -15.1 & -14.6 \\
$\mathrm{Mg}^{2+}$ & -104.5 & -104.2 & -105.7 & -114.2 & -112.7 & -117.7 & -114.5 \\
$\mathrm{Ca}^{2+}$ & -60.2 & -59.6 & -59.8 & -64.8 & -64.6 & -67.6 & -66.1 \\
\hline & & & & & & \\
& & & & & \\
$\mathrm{Li}^{+}$ & -37.5 & -37.2 & -39.8 & -40.5 & -39.6 & -41.0 & -41.0 \\
$\mathrm{Na}^{+}$ & -24.8 & -24.5 & -26.1 & -27.0 & -26.2 & -27.8 & -27.3 \\
$\mathrm{~K}^{+}$ & -18.2 & -18.0 & -17.7 & -18.2 & -17.9 & -19.3 & -19.0 \\
$\mathrm{Mg}^{2+}$ & -113.4 & -113.1 & -116.5 & -128.3 & -124.3 & -130.9 & -125.8 \\
$\mathrm{Ca}^{2+}$ & -69.4 & -68.8 & -70.4 & -73.3 & -73.2 & -75.6 & -74.7 \\
\hline \hline
\end{tabular}

${ }^{\mathrm{a}}$ The BSSE was corrected using the CP method.

${ }^{\mathrm{b}}$ All calculations were carried out using the $6-31+\mathrm{G}(d, p)$ basis set.

TABLE III. Optimized intermolecular parameters (in $\AA$ ) for charge transfer complexes $M \cdots$ thiophene and $M \cdots$ pyrrole. The parameters are defined in Fig. 1.

\begin{tabular}{|c|c|c|c|c|c|c|c|c|c|c|}
\hline \multicolumn{6}{|c|}{$M \cdots$ Thp } & \multicolumn{5}{|c|}{$M \cdots \mathrm{Py}$} \\
\hline & $\mathrm{Li}$ & $\mathrm{Na}$ & K & $\mathrm{Mg}$ & $\begin{array}{l}\mathrm{Ca} \\
\mathrm{MP} 2\end{array}$ & $\mathrm{Li}$ & $\mathrm{Na}$ & K & $\mathrm{Mg}$ & $\mathrm{Ca}$ \\
\hline$d_{1}$ & 2.572 & 3.660 & 3.834 & 3.875 & 4.063 & 2.608 & 3.168 & 3.284 & 3.423 & 3.458 \\
\hline$d^{2,5}$ & 2.151 & 3.512 & 3.644 & 3.777 & 3.935 & 2.581 & 3.144 & 3.316 & 3.455 & 3.467 \\
\hline$d^{3,4}$ & 2.222 & 3.425 & 3.510 & 3.739 & $\begin{array}{r}3.859 \\
\mathrm{HF}\end{array}$ & 2.521 & 3.089 & 3.342 & 3.505 & 3.483 \\
\hline$d_{1}$ & 2.515 & 5.876 & 6.090 & 5.679 & 6.501 & 2.825 & 3.497 & 3.646 & 4.970 & 5.331 \\
\hline$d^{2,5}$ & 2.161 & 5.872 & 6.098 & 5.645 & 6.471 & 2.742 & 3.429 & 3.620 & 5.003 & 5.316 \\
\hline$d^{3,4}$ & 2.198 & 5.906 & 6.145 & 5.649 & $\begin{array}{l}6.476 \\
\text { B3LYP }\end{array}$ & 2.580 & 3.299 & 3.554 & 5.050 & 5.283 \\
\hline$d_{1}$ & 2.551 & 4.122 & 4.339 & 5.720 & 5.841 & 2.600 & 3.324 & 3.558 & 3.937 & 3.974 \\
\hline$d^{2,5}$ & 2.143 & 4.021 & 4.217 & 5.548 & 5.687 & 2.557 & 3.282 & 3.560 & 3.977 & 3.987 \\
\hline$d^{3,4}$ & 2.167 & 3.973 & 4.143 & 5.406 & 5.564 & 2.492 & 3.207 & 3.545 & 4.037 & 4.005 \\
\hline \multicolumn{11}{|c|}{ B3PW91 } \\
\hline$d_{1}$ & 2.545 & 3.918 & 4.193 & 4.024 & 4.268 & 2.618 & 3.287 & 3.508 & 3.507 & 3.617 \\
\hline$d^{2,5}$ & 2.142 & 3.852 & 4.054 & 3.964 & 4.200 & 2.572 & 3.251 & 3.516 & 3.456 & 3.620 \\
\hline$d^{3,4}$ & 2.164 & 3.713 & 3.963 & 3.960 & $\begin{array}{r}4.180 \\
\text { PBE }\end{array}$ & 2.501 & 3.184 & 3.481 & 3.572 & 3.626 \\
\hline$d_{1}$ & 2.587 & 3.703 & 3.909 & 3.850 & 4.118 & 2.532 & 3.184 & 3.414 & 3.431 & 3.545 \\
\hline$d^{2,5}$ & 2.170 & 3.541 & 3.739 & 3.778 & 4.025 & 2.491 & 3.152 & 3.424 & 3.461 & 3.554 \\
\hline$d^{3,4}$ & 2.176 & 3.423 & 3.583 & 3.767 & 3.986 & 2.451 & 3.099 & 3.432 & 3.513 & 3.583 \\
\hline \multicolumn{11}{|c|}{ MPW1PW91 } \\
\hline$d_{1}$ & 2.538 & 3.772 & 4.019 & 3.900 & 4.156 & 2.581 & 3.218 & 3.437 & 3.427 & 3.550 \\
\hline$d^{2,5}$ & 2.136 & 3.628 & 3.860 & 3.830 & 4.070 & 2.543 & 3.177 & 3.436 & 3.456 & 3.553 \\
\hline$d^{3,4}$ & 2.160 & 3.537 & 3.748 & 3.816 & 4.036 & 2.481 & 3.108 & 3.421 & 3.505 & 3.557 \\
\hline
\end{tabular}


TABLE IV. Optimized intermolecular parameters (in $\AA$ ) for charge transfer complexes $M^{n+} \cdot$. thiophene and $M^{n+}$...pyrrole. The parameters are defined in Fig. 1.

\begin{tabular}{|c|c|c|c|c|c|c|c|c|c|c|}
\hline \multicolumn{6}{|c|}{$M^{n+} \ldots$ Thp } & \multicolumn{5}{|c|}{$M^{n+} \ldots \mathrm{Py}$} \\
\hline & $\mathrm{Li}^{+}$ & $\mathrm{Na}^{+}$ & $\mathrm{K}^{+}$ & $\mathrm{Mg}^{2+}$ & $\begin{array}{l}\mathrm{Ca}^{2+} \\
\mathrm{MP} 2\end{array}$ & $\mathrm{Li}^{+}$ & $\mathrm{Na}^{+}$ & $\mathrm{K}^{+}$ & $\mathrm{Mg}^{2+}$ & $\mathrm{Ca}^{2+}$ \\
\hline$d_{1}$ & 2.548 & 2.967 & 3.391 & 2.582 & 3.017 & 2.298 & 2.868 & 3.148 & 2.557 & 2.794 \\
\hline$d^{2,5}$ & 2.383 & 2.809 & 3.232 & 2.405 & 2.843 & 2.324 & 2.816 & 3.145 & 2.483 & 2.808 \\
\hline$d^{3,4}$ & 2.352 & 2.767 & 3.171 & 2.389 & $\begin{array}{r}2.815 \\
\mathrm{HF}\end{array}$ & 2.296 & 2.674 & 3.073 & 2.291 & 2.747 \\
\hline$d_{1}$ & 2.512 & 2.941 & 3.416 & 2.536 & 2.977 & 2.243 & 2.860 & 3.288 & 2.530 & 2.783 \\
\hline$d^{2,5}$ & 2.394 & 2.848 & 3.352 & 2.421 & 2.885 & 2.297 & 2.819 & 3.267 & 2.479 & 2.826 \\
\hline$d^{3,4}$ & 2.390 & 2.851 & 3.372 & 2.433 & $\begin{array}{l}2.905 \\
\text { B3LYP }\end{array}$ & 2.304 & 2.688 & 3.172 & 2.301 & 2.786 \\
\hline$d_{1}$ & 2.511 & 2.939 & 3.377 & 2.561 & 2.975 & 2.237 & 2.918 & 3.245 & 3.271 & 2.806 \\
\hline$d^{2,5}$ & 2.352 & 2.787 & 3.255 & 2.397 & 2.833 & 2.273 & 2.823 & 3.203 & 2.950 & 2.808 \\
\hline$d^{3,4}$ & 2.322 & 2.743 & 3.226 & 2.382 & 2.826 & 2.262 & 2.615 & 3.084 & 2.252 & 2.730 \\
\hline \multicolumn{11}{|c|}{ B3PW91 } \\
\hline$d_{1}$ & 2.519 & 2.952 & 3.376 & 2.557 & 2.966 & 2.263 & 2.926 & 3.246 & 3.159 & 2.792 \\
\hline$d^{2,5}$ & 2.358 & 2.795 & 3.245 & 2.392 & 2.818 & 2.286 & 2.831 & 3.200 & 2.867 & 2.793 \\
\hline$d^{3,4}$ & 2.324 & 2.745 & 3.207 & 2.375 & 2.807 & 2.260 & 2.620 & 3.069 & 2.242 & 2.715 \\
\hline \multicolumn{11}{|c|}{ PBE } \\
\hline$d_{1}$ & 2.517 & 2.947 & 3.370 & 2.568 & 2.976 & 2.256 & 2.931 & 3.235 & 3.288 & 2.818 \\
\hline$d^{2,5}$ & 2.347 & 2.778 & 3.230 & 2.393 & 2.818 & 2.289 & 2.828 & 3.186 & 2.962 & 2.805 \\
\hline$d^{3,4}$ & 2.309 & 2.721 & 3.186 & 2.371 & 2.801 & 2.252 & 2.607 & 3.057 & 2.252 & 2.711 \\
\hline \multicolumn{11}{|c|}{ MPW1PW91 } \\
\hline$d_{1}$ & 2.509 & 2.933 & 3.355 & 2.551 & 2.958 & 2.242 & 2.882 & 3.211 & 3.119 & 2.782 \\
\hline$d^{2,5}$ & 2.348 & 2.779 & 3.226 & 2.387 & 2.812 & 2.297 & 2.801 & 3.171 & 2.838 & 2.787 \\
\hline$d^{3,4}$ & 2.315 & 2.731 & 3.190 & 2.371 & 2.801 & 2.254 & 2.614 & 3.057 & 2.238 & 2.713 \\
\hline
\end{tabular}

\section{B. Geometries}

The intermolecular parameters used to compare the geometries predicted by the different methods for the ten complexes investigated consist of three distances $d_{1}, d^{2,5}$, and $d^{3,4}$, which are defined in Fig. 1. It should be noted that we are interested in how the strength predicted by the different methods for the $M \cdots \pi$ and $M^{n+} \cdots \pi$ interactions correlates with the geometry of the complexes, this information being satisfactorily described by the three parameters considered. Thus other intermolecular parameters as angles or dihedrals, which can be supplied upon request to authors, are not essential in this case because the relative arrangement between the two fragments of the complex was the same in all cases, i.e., the $M$ or $M^{n+}$ specie above the $\pi$-system of the heterocycle.

Table III summarizes the optimized geometric parameters for the ten charge transfer complexes. The geometries obtained using the MP2 method are fully consistent with the interaction energies predicted at the same computational level. Thus the three distances increase with the size of $M$ for both $M \cdots$ Thp and $M \cdots$ Py complexes, and for a given $M$ the distances are larger for $M \cdots$ Thp complexes than for $M \cdots \mathrm{Py}$ complexes. Furthermore, as expected for a weak interaction, the parameters are relatively large ranging from 2.151 to
$4.063 \AA$ for $M \cdots$ Thp and from 2.521 to $3.483 \AA$ for $M \cdots$ Py.

On the other hand, the HF parameters reflect the limitations of this method to describe charge transfer complexes. Thus the resulting HF intermolecular distances show that, in many cases, geometry optimizations led to the disruption of the complex. The four DFT methods tend to overestimate the intermolecular distances with respect to the MP2 values. The largest overestimation was obtained at the B3LYP level. Substitution of the LYP functional by the PW91 one to express correlation induces an improvement in the geometries. As expected, the PBE and MPW1MPW91 provide the best DFT results, the intermolecular distances obtained at these levels being $\sim 0.1 \AA$ greater than the MP2 ones. The results are in excellent agreement with the interaction energies displayed in Table I.

Table IV lists the optimized geometric parameters for $M^{n+} \ldots$ Thp and $M^{n+}$. Py complexes. Comparison between the distances predicted for $M \cdots \pi$ and $M^{n+\cdots} \pi$ at the MP2 level indicates a shortening of $\sim 0.3$ and $\sim 1.0 \AA$ for $M^{+}$and $M^{2+}$, respectively. On the other hand, the geometries calculated at the different levels for $M^{n+} \ldots$ Thp and $M^{n+} \cdots$ Py complexes show a remarkable agreement. Thus HF and DFT methods are able to describe satisfactorily the geometry of the electrostatically stabilized complexes. Accordingly, although the shortcomings of the exchange functionals pro- 
TABLE V. BSSE-uncorrected interaction energies ${ }^{\mathrm{a}}$ (in $\mathrm{kcal} / \mathrm{mol}$ ) calculated for charge transfer complexes $M \cdots$ thiophene and $M \cdots$ pyrrole. In every case the level of theory used to calculate the interaction energy is identical to that used to obtain the optimized geometry with the exception of the MP4 level, where the MP2 optimized geometry was used (see Sec. II). ${ }^{\text {a }}$

\begin{tabular}{crrrrrrr}
\hline \hline$M$ & MP2 & MP4 & HF & B3LYP & B3PW91 & PBE & MPW1PW91 \\
\hline \multicolumn{7}{c}{$M \cdots$ Thiophene } \\
$\mathrm{Li}$ & -15.8 & -13.1 & -9.3 & -20.5 & -21.4 & -20.3 & -21.5 \\
$\mathrm{Na}$ & -2.1 & -2.3 & 0.0 & -0.4 & -0.6 & -1.7 & -1.2 \\
$\mathrm{~K}$ & -2.9 & -2.9 & 0.0 & -0.4 & -0.5 & -1.9 & -1.2 \\
$\mathrm{Mg}$ & -2.6 & -2.2 & -0.1 & 0.0 & -0.2 & -1.5 & -0.9 \\
$\mathrm{Ca}$ & -3.0 & -3.6 & 0.0 & -0.6 & -0.3 & -1.7 & -1.5 \\
\hline & & & \multicolumn{7}{c}{$M \cdots$ Pyrrole } & & \\
$\mathrm{Li}$ & -7.0 & -7.3 & -2.9 & -5.0 & -5.3 & -7.5 & -6.5 \\
$\mathrm{Na}$ & -3.9 & -4.2 & -2.3 & -1.9 & -2.2 & -3.6 & -3.0 \\
$\mathrm{~K}$ & -5.4 & -5.3 & -1.8 & -2.0 & -2.3 & -3.8 & -3.3 \\
$\mathrm{Mg}$ & -3.4 & -3.0 & -0.1 & -0.3 & -1.0 & -2.6 & -1.9 \\
$\mathrm{Ca}$ & -5.4 & -4.9 & -0.2 & -1.2 & -1.7 & -3.5 & -3.2 \\
\hline \hline
\end{tabular}

$\overline{\bar{a} \text { All calculations were carried out using the } 6-31+\mathrm{G}(d, p) \text { basis set. }}$

duced an overestimation of the corresponding interaction energies (Table II), they do not affect the geometries.

\section{Analysis of the basis set superposition error}

The importance of the BSSE in the computed interaction energies has been calibrated by comparing the results displayed in Tables I and II with those listed in Tables V and VI, which show the interaction energies calculated without applying the $\mathrm{CP}$ correction. We are aware that the $\mathrm{CP}$ method provides only approximated results since it overestimates the size of the BSSE. However, early studies on van der Waals and hydrogen bonded complexes have shown that the $\mathrm{CP}$ correction provides interaction energies within the experimental uncertainty [28-30].

Inspection to the results obtained for $M \cdots$ Thp and $M \cdots$ Py complexes indicates that the BSSE plays a similar but important role in MP2 and MP4 calculations. Thus the BSSE ranges from 1.2 to $4.0 \mathrm{kcal} / \mathrm{mol}$ for $M=\mathrm{Li}, \mathrm{Na}$, and $\mathrm{K}$ and from 0.9 to $2.9 \mathrm{kcal} / \mathrm{mol}$ for $M=\mathrm{Mg}$ and $\mathrm{Ca}$, these values being about $32-67 \%$ of the interaction energy. DFT methods seem to be less susceptible to the basis set truncation than conventional MP $n$ methods for $M \cdots \pi$ complexes. However, this conclusion must be taken with caution since, as was shown above, these complexes are poorly described by DFT methods, especially by B3LYP and B3PW91. The BSSE of the PBE and MPW1PW91 functionals, which provide the more reliable DFT results for $M \cdots \pi$ complexes, range from 0.1 to $0.8 \mathrm{kcal} / \mathrm{mol}$ that corresponds to less than $22 \%$ of the interaction energy. Similarly, the BSSE predicted by the four functionals investigated for $\mathrm{Li} \cdot \cdots$ Thp and $\mathrm{Li} \cdot$ Py complexes was lower than $12 \%$ of the interaction energy. The overall of these results suggest that, in spite of their limitations, the DFT methods are less sensitive to BSSE than conventional MP2 and MP4 methods. However, the magnitude of the BSSE oscillates with the different functionals.

The uncorrected interaction energies calculated for $M^{n+}$. Thp and $M^{n+}$. Py at the ab initio MP2, MP4, and HF levels (Table VI) reveal that the BSSE is considerably smaller than for $M \cdots \pi$ complexes. Moreover, the size of the BSSE decreases when the electrostatic component of the interaction increases. Thus the BSSE is smaller for $M^{n+} \ldots$ Thp than for $M^{n+} \ldots$ Py and, within each group, the influence of the basis set truncation is smaller for $M^{2+}$ than for $\mathrm{M}^{+}$-containing complexes. Examination of the DFT results indicates that, independently of the functional, the BSSE is almost negligible for the electrostatically stabilized complexes. Thus the BSSE is around 2-4\% for the complexes

TABLE VI. BSSE-uncorrected interaction energies ${ }^{\mathrm{a}}$ (in $\mathrm{kcal} / \mathrm{mol}$ ) calculated for charge transfer complexes $M^{n+}$...thiophene and $M^{n+}$.. pyrrole. In every case the level of theory used to calculate the interaction energy is identical to that used to obtain the optimized geometry with exception of the MP4 level, where the MP2 optimized geometry was used (see Sec. II). ${ }^{\mathrm{a}}$

\begin{tabular}{cccccccc}
\hline \hline$M^{n+}$ & MP2 & MP4 & HF & B3LYP & B3PW91 & PBE & MPW1PW91 \\
\hline \multicolumn{7}{c}{$M^{n+.}$ Thiophene } \\
$\mathrm{Li}^{+}$ & -35.1 & -34.7 & -32.6 & -35.0 & -34.1 & -36.0 & -35.4 \\
$\mathrm{Na}^{+}$ & -22.7 & -22.5 & -20.8 & -22.5 & -21.6 & -23.5 & -20.8 \\
$\mathrm{~K}^{+}$ & -16.2 & -14.4 & -13.3 & -14.2 & -13.9 & -15.5 & -15.0 \\
$\mathrm{Mg}^{2+}$ & -110.2 & -110.0 & -106.3 & -115.0 & -113.5 & -118.7 & -115.5 \\
$\mathrm{Ca}^{2+}$ & -64.5 & -64.2 & -60.6 & -65.4 & -65.1 & -68.2 & -66.6 \\
\hline & & & & & \\
& & & & & \\
$\mathrm{Li}^{+}$ & -40.5 & -40.1 & -40.2 & -41.1 & -40.2 & -41.8 & -41.6 \\
$\mathrm{Na}^{+}$ & -27.2 & -27.0 & -26.6 & -27.5 & -26.7 & -28.3 & -27.9 \\
$\mathrm{~K}^{+}$ & -20.9 & -20.7 & -17.8 & -18.5 & -18.2 & -19.7 & -19.3 \\
$\mathrm{Mg}^{2+}$ & -117.6 & -117.5 & -117.1 & -128.8 & -124.8 & -131.4 & -126.3 \\
$\mathrm{Ca}^{2+}$ & -73.4 & -73.2 & -70.8 & -73.9 & -73.8 & -76.3 & -75.3 \\
\hline \hline
\end{tabular}

${ }^{\mathrm{a}}$ All calculations were carried out using the $6-31+\mathrm{G}(d, p)$ basis set. 
formed with $\mathrm{M}^{+}$and smaller than $1 \%$ for those containing $\mathrm{M}^{2+}$.

\section{CONCLUSIONS}

In this work we investigated $M \cdots \pi$ and $M^{n+} \cdots \pi$ complexes using DFT and ab initio methods. The performance of four DFT functionals (B3LYP, B3PW91, PBE, and MPW1PW91) was tested considering 20 different complexes. Comparison with MP2 and MP4 results indicated that the B3LYP and B3PW91 methods are completely inadequate in prediction of $M \cdots \pi$ interaction energy and geometry. However, the MPW1PW91 functional provided a good description of all the charge transfer complexes investigated. Furthermore, this DFT method predicts reliable interaction energies even when the molecular geometries were optimized using a different computational procedure. The BSSE estimated for $M \cdots$ Thp and $M \cdots$ Py was found to be consid- erably smaller for DFT methods than for conventional $a b$ initio ones. Interestingly, the failure of the B3LYP and B3PW91 was also reported for electron donor acceptor systems formed from ethylene interacting with a halogen molecule [31], where the interaction is expected to be weaker than in the $M \cdots \pi$ complexes presented in this work.

On the other hand, electrostatically stabilized $M^{n+\cdots} \pi$ complexes are rightly described by all the methods considered in this work, although DFT methods overestimate the strength of the interaction with respect to the ab initio ones. In general the size of the BSSE was found to be small in $M^{n+} \ldots$ Thp and $M^{n+} \ldots$ Py complexes, being almost negligible for the four DFT methods.

\section{ACKNOWLEDGMENTS}

The authors are indebted to CESCA (Centre de Supercomputació de Catalunya), CEPBA (Centre Europeu de Paral-lelisme de Barcelona), and MCYT (MAT2003-00251).
[1] J.-L. Brédas, J. G. Thémans, J. G. Fripiat, J. M. André, and R. R. Chance, Phys. Rev. B 29, 6761 (1984).

[2] M. Fahlman, D. Beljonne, M. Löglund, R. H. Friend, A. B. Holmes, J.-L Brédas, and W. R. Salaneck, Chem. Phys. Lett. 214, 327 (1993).

[3] C. Fredriksson and S. Stafström, J. Chem. Phys. 101, 9137 (1994).

[4] S. Irle and H. Lischka, J. Chem. Phys. 103, 1508 (1995).

[5] T. Tamm, J. Tamm, and M. Karelson, Int. J. Quantum Chem. 88, 296 (2002).

[6] J.-L. Brédas, Adv. Mater. (Weinheim, Ger.) 7, 263 (1995).

[7] C. Alemán, D. Curcó, and J. Casanovas, Chem. Phys. Lett. 383, 408 (2004).

[8] J. J. Novoa and C. Sosa, J. Phys. Chem. 99, 15837 (1995).

[9] H. Guo, N. Gresh, B. Roques, and D. R. Salahub, J. Phys. Chem. B 104, 9746 (2000).

[10] J. Sponer, J. Leszczynski, and P. Hobza, J. Phys. Chem. 100, 1965 (1996).

[11] O. Lukin and J. Leszczynski, J. Phys. Chem. A 106, 6775 (2002).

[12] T. A. Wesolowski, O. Parisel, Y. Ellinger, and J. Weber, J. Phys. Chem. A 101, 7818 (1997).

[13] Y. He, J. Grafenstein, E. Kraka, and D. Cremer, Mol. Phys. 98, 1639 (2000).

[14] A. K. Chandra and M. T. Nguyen, Chem. Phys. 232, 299 (1998)

[15] X. Ye, Z.-H. Li, W. Wang, K. Fan, W. Xu, and Z. Hua, Chem.
Phys. Lett. 397, 56 (2004).

[16] J. Sponer, J. Leszczynski, and P. Hobza, J. Comput. Chem. 17, 841 (1996).

[17] M. Elstner, P. Hobza, T. Frauenheim, S. Suhai, and E. Kaxiros, J. Chem. Phys. 114, 5149 (2001).

[18] GAUSSIAN 98, Revision A.7, M. J. Frisch et al., Gaussian, Inc., Pittsburgh, PA, 1998.

[19] C. Møller and M. S. Plesset, Phys. Rev. 46, 618 (1934).

[20] A. D. Becke, J. Chem. Phys. 98, 1372 (1993).

[21] C. Lee, W. Yang, and R. G. Parr, Phys. Rev. B 37, 785 (1993).

[22] .J. P. Perdew and Y. Wang, Phys. Rev. B 45, 13244 (1992).

[23] J. P. Perdew, K. Burke, and M. Ernzerhof, Phys. Rev. Lett. 77, 3865 (1996).

[24] J. P. Perdew, K. Burke, and M. Ernzerhof, Phys. Rev. Lett. 78, 1396 (1997).

[25] C. Adamo and V. Barone, J. Chem. Phys. 108, 664 (1998).

[26] M. J. Frich, J. A. Pople, R. Krishnam, and J. S. Binkley, J. Chem. Phys. 80, 3264 (1984).

[27] S. F. Boys and F. Bernardi, Mol. Phys. 19, 553 (1970).

[28] M. Gutowski, J. G. C. M. van Duijneveldt-van de Rijdt, J. H. van lente, and F. B. van Duijneveldt, J. Chem. Phys. 98, 4728 (1993).

[29] D. Feller, J. Chem. Phys. 96, 6104 (1992).

[30] J. J. Novoa, M. Planas, and M.-H. Whangbo, Chem. Phys. Lett. 225, 240 (1994).

[31] E. Ruiz, D. R. Salahub, and A. Vela, J. Phys. Chem. 100, 12265 (1996). 